\title{
Obesity and circadian system
}

\begin{abstract}
Obesity, an epidemic health concern that related with high levels of worldwide morbidity and mortality levels, showed marked increase to lastyears. Studies focus on the factors leading such rise to make effective and long lasting intervensions intended for obesity. Decreased nightly sleep duration,compared to the past century, is considered as one of these factors and studies emphasize the relation between changing sleep patterns and obesity and accompanying health disorders as impaired glucose metabolism, cardiovascular diseases, type 2 diabetes mellitus, hypertension those also have high prevelances. This literature review is prepared with the articles those taken from Pubmed and Science Direct especially were published after 2010 to comply with the aim of demonstrating the association of sleep with obesity and mentioned health disorders, basic mechanisms undelying this association and factors affecting the functioning of these mechanism.
\end{abstract}

Volume 6 Issue I - 2017

\author{
Sehnaz Ayar, Binnur Okan Bakir \\ Department of Nutrition and Dietetics, Yeditepe University, \\ Turkey
}

Correspondence: Binnur Okan Bakir, Department of Nutrition and Dietetics, Yeditepe University, Inonü Mah. Kayișdaği Cad. 34755 Atașehir/ Istanbul,Turkey,Tel 00902165780657 , Fax 00902165780496, Email binnur.bakir@yeditepe.edu.tr

Received: November 04, 2016 | Published: January 24, 2017

Keywords: sleep, sleep loss, obesity, obesity comorbidities, circadian system, hormones, metabolism, sleep induced changes

Abbreviations: NPY, neuropeptide y; BMI, body mass index; SWS, slow wave sleep; NREM, non-rapid eye movemenT; RVLM, rostro ventrolateral medulla; $\mathrm{TSH}$, thiroid stimulating hormone

\section{Introduction}

Obesity, a chronic inflammatuar state, is one of the most significant health problem that characterized by abnormal fat accumulation in the body and increasing ratio among fatty and lean body masses thereby rising body mass index (BMI). According to world health organization(WHO) statistics in 2014, 1.9billion adults were overweight $\left(\mathrm{BMI}>25 \mathrm{~kg} / \mathrm{m}^{2}\right)$ and 600 millions of them were obese $\left(\mathrm{BMI}>30 \mathrm{~kg} / \mathrm{m}^{2}\right)$. Obesity can be handled as an epidemic and life threatening issue with its folding rates from the past to the present and accompanied health disorders such as type 2 diabetes mellitus, cancer, cardiovascular diseases, atherosclerosis, hypertension, hypertrygliceridemia etc. This issue with its comorbidities became a global concern due to its doubling prevelance rates between 1980 and 2014 independent from the welfare or development level of country or age, sex, and race. ${ }^{1}$ In the same way, TURDEP statistics in 2010 represents the increased percentages of obese and overweight individuals compared to 1997 and 1998 in Turkey. ${ }^{2}$ Changing life styles and consumption patterns, reasoned from industrialization, leads increasing rates of obesity. Along with industrialization, physical works has reduced whereas the time people spent on sitting works has increased. This change in working style leads people to have more sedantary life routines that can be illustrated as one of the main reason of obesity. In addition to alterations on working styles, extension of working times also affect the diet and life patterns. Increasing workinghours reduce the chance for consumption of home made foods and cause people to consume packaged, ready to consume food that found to be responsible from increasing daily energy intake and rising obesity levels related with abdominal fat accumulation. ${ }^{3}$ Besides, increasing mass media marketing blaze the trail to consumption of high energy dense food having poor nutritional values that especially responsible for childhood obesity ${ }^{4}$ because of childs vulnerability to marketing. ${ }^{5}$

Increasing worldwide focus on obesity is reasoned from its rising and life threatening comorbidities rather than obesity itself. So, any issue on the basis of metabolism, hormones or nervous system which have considered as being precursor or risk factor of obesity and its comorbidities have began to studied to detect causes and thereby making well functioning interventions to prevent such health problems. Herein, another crucial and recently quiteunder investigated issue about development of obesity is changing sleeep patterns that can be seen among all age groups as independent from, sex, race and socioeconomic status.

The relation between sleep and obesity and accompanied health disorders has became one of the recently most studied issue owing tochanging sleep patterns along with the time has occured simultaneous with rising prevelance of obesity. ${ }^{6}$ Statistics revealed 6.8hours of nightly sleep for Western Societies as lower than past centuries. ${ }^{7}$ In behavioural risk factor surveillence system study conducted in 12 state of USA, aproximetelly $\% 35$ of adults have been reported as sleep less than 7hours without any difference related with gender. ${ }^{8}$ In adittion, increasing percentage of short sleepers(less than 7hours) have been reported among American adolescents. ${ }^{9}$ Sleep has been investigated within its several components,such as sleep durations, sleep patterns (timing, regularity), sleep architecture and sleep quality, those revealing metabolic, hormonal and neural effects as a result of human and animal experiments. Because of strong relationship being detected between obesity and sleep variables with the contribution of many conducted correlational or experimental researches, the need for adding sleep intervention to obesity prevention programs has been defended. ${ }^{10}$ Decreased sleephours from the past to the century ${ }^{11}$ and changing sleep patterns in terms of time and regularity have led alterations on energy intake ${ }^{12}$ changing food patterrns ${ }^{13-15}$ and meal timing ${ }^{16}$ and also alterations on bodily circadian mechanism those have found to be precessor of obesity related hormonal ${ }^{17}$ and metabolic changes, ${ }^{18,11}$ unhealthy eating behaviours ${ }^{19}$ and thereby increase in obesity indicators ${ }^{20}$ both for adults ${ }^{6}$ and children..$^{14,15}$ Studies also revealed strong relationship between changes in such mentioned sleep variables and rising levels of chronic diseases as diabetes mellitus, cardiovascular diseases and hypertension., ${ }^{721}$ The purpose of this review to agglomerate evidences support the role of sleep variables and circadian clock in the development of obesity and metabolic diseases and lighten intermediary factors on mentioned relationships to inspire government in the development of well functioning health 
policies on such interceptible area for prevention and treatment of obesity and metabolic diseases those take a big cost from national health budget.

\section{Discussion}

\section{Sleep duration and obesity}

It has been indicated that nightly sleep durations have been declined historically from childhood ages to adolescence altough it varies among sex, race and socioeconomic status Keyes et al. ${ }^{9}$ One of the recent research on sleep durations has revealed that the average nightly sleep duration has reduced by 1.5 hours compared to past century. ${ }^{11}$ Such change in sleep durations over the centurieshas been thought to leading obesity by several aspects such as increasing energy intake. ${ }^{12}$ changing food patterns, ${ }^{13,14}$ increasing choice of sugary and more energy dense foods and beverages,${ }^{15}$ changes in meal timing, ${ }^{16}$ and changes in hormones, ${ }^{17}$ and metabolism. ${ }^{18}$

Most of the studies has detected the U-shaped relationship between sleep duration and basic obesity measures like waisthip circumferences, body mass index, body weightand body fat deposition that means both short and long durations of nightly sleep leads rising obesity levels from the aspects of measured obesity indicators. A recently conducted experiment among childrens aged from 8 to 10 revealed significant relation in terms of increased BMI and body fat deposition both for short and long time sleepers and found increasing waist circumferences only related with short sleep durations as independent from caloric intake and physical activity level, ${ }^{14}$ while another study examining adults found increase in BMI scores related with both short and long sleep durations only for female participants and found $\mathrm{L}$ shaped relation for males meaning only short sleep durations have been found as related with rising BMI scores. ${ }^{6}$ Increase in caloric intake especially those from fat, increasing portion sizes, and late time nightly eatings and increasing total number of meals have been found as significantly correlated with body weight increase during sleep restriction days particularly among females and African American people ${ }^{16}$ in contrast to NHANES being conducted between 2005 and 2010 among American people has found increase on energy intake after 20:00hours despite of stable 24hours calories amount across short and long sleepers. ${ }^{19}$ The change in dietary patterns is seen as another touchstone mediating the obesity and sleep relationship. A study completed by examining the long term(200days) sleep and related diet habits of Danish school children demonstrated every 15 minutes sleep restriction as responsible from 0.3 increase on percentage of sugar sweetened beverages and 1.2 increase on percentage of additional sugar to diet among the total daily caloric intake in addition to indication of increasing 134 calories in daily caloric intake during short sleep period compared to long sleep period..$^{15}$

\section{Sleep patterns and obesity}

The times people spent on screening, social media and working affect not only sleep durations but also its regularity and timing. In a study, sleep patterns determined by sleep times on weekend and weekdays have been associated with waist and hip circumferences and BMI for children and adolescents. ${ }^{20}$ In the study revealingvariabilty in sleep durations as highly correlated with higher BMI scores and low self reported physical activity status for both sexes, the positive association between sleep timing variability(sleep regularity) and obesity related health problems such as diabetes and coronary heart diseases also have been mentioned for both male and female adults. ${ }^{21}$
Late meal times presented as one of the peacemakers in the realation of sleep timing and obesity. Late sleep times probably leads delay on meal times proposed as related with weight loss effectivenes. ${ }^{22}$ In a study that aimed to look on relation between elevated BMI and sleep timing, late sleepers had demonstrate tendency toward cumulative caloric intake as taking most of the calories at dinner and after $8 \mathrm{pm}$ that resulted in consumption of 248 more calories in comparison with early sleepers. Besides, sleep timing induced food patterns among late sleepers as higher fast food and full energy beverages consumption acompanied by lower fruits and vegetables consumptions have been related with rising obesity levels..$^{23}$ Late sleep times also affect breakfast frequencies ${ }^{24}$ as one of the predictor of metabolic syndrome ${ }^{25}$ and lead increase caloric intake in nighthours which have been proved as disrupt the circadian clock as another obesity pathway that will be mentioned later.

\section{Sleep architecture and obesity}

The American Academy of Sleep divides sleep into four categories as three stages of non-rapid eye movement (NREM) sleep namely N1, N2, N3 also called as slow wave sleep (SWS) and rapid eye movement (REM) sleep. In recentyears increasing attention have started through sleep architecture due to several studies that found sleep stages, particularly N3 sleep and REM sleep as associated with body weight, ${ }^{26}$ glucose metabolism, ${ }^{27}$ changing brain signals, metabolic rate and hunger. ${ }^{28}$ In a study that found N3 sleep duration and its percentage in total sleep duration as important in terms of insulin secretion, increasing parasympathetic activity in $\mathrm{N} 3$ sleep have been found related with improving insulin secretions in obese and overweight adolescents while the N2 sleep negatively correlated with insulin sensitivity. ${ }^{29}$ In one of the previous research that has revealed decreased percentage of REM and stage 2 sleep in spite of increased SWS percentage in total sleep duration after sleep restriction, stage 2 sleep has been found as positively related with increased RMR and inversely related with desire for salty and sweet foods and REM sleep showed inverse associations with hunger rates, fat and carbohydrate intake. ${ }^{28}$ In a study revealing the effect of REM sleep on energy and hunger mechanism, REM sleep deprivated rates have demonstrated decreased weight inspite of upregulation of neuropeptide Y(NPY) and downregulation of proopiomelanocortin(POMC) in hypothalamic arc as favoring hyperphagia. ${ }^{30}$ Tasali et al..$^{27}$ have reported decreased glucose tolerance and increased risk of type 2 diabetes mellitus with stable insulin secretion in spite of lowered insulin sensitivity as a result of experimental SWS suppresion without any interference with total sleep time. ${ }^{27}$

\section{Sleep quality and obesity}

Decreasing sleep quality is another aspect of view which have been found as correlated with increasing obesity measures like bodily fat depositions, weight and waist-hip ratio ${ }^{20}$ and impaired glucose homeostasis in terms of its mediation in sleep and obesity affair. One of the laboratory study that performed as exposing rats to sleep fragmentation by disturbing the wake-sleep cycles, thereby correpted sleep quality, in addition to sleep restriction(4 hour sleep per night for eight nights), found weight and metabolic responsesbeing given to sleep fragmentations similar to those obtained by the effect of sleep restriction. To data obtained from aforementionadstudy, the blood insulin were lower while the glucose was higher as response to intravenous glucose tolerance test in both groups, compared to the values reported before the experiment. In addition torising plasma glucose and decreasing plasma insulin levels displaying the impact 
of sleep quality on the development of hyperglycemia, decreasing corticosterone levels and similar body weight change have been recorded with no observed change in energy intake as a result of sleep restriction and sleep fragmentation ${ }^{31}$ in contrast to the Wang et al. ${ }^{32}$ those had explained the relation between decreasing sleep quality and body weight from the aspect of increasing energy intake as an obesogenic behaviour reasoned from leptin resistance and also found low sleep quality as responsible for growing visceral and subcutanous bodily fat depots ${ }^{32}$ Another study tested the impact of sleep duration and sleep quality on glucose homeostasis and glucose tolerance had found 1 hour increase in sleep quality related with improvement on both peripheral and hepatic insulin sensitivity and one percent decline in insulin secretion. ${ }^{33}$

\section{Circadian rhythm and obesity}

Circadian clock: The circadian rhythm has became another important issue as an obesity pathway because of its effects on energy balance and glucose metabolism being proved by several researches. The circadian system is an endogenous 24 hours repetitive mechanism work on regulating bodily hormonal, neural and metabolic activities within the external cues such as daytime light exposure and food availability as most important ones to catch optimal physical homeostasis level for survival. In mammals there present two main administrator of circadian clock namely, the Master Clock, located in subrachiasmic nucleus in front of the hypothalamus, ensure homeostasis between environmental cues and physiology and the Slave clock, the name given to all peripheral circadian oscillators, work through the neural and hormonal informations from Master clock. Every hormonal and metabolic activities have their own diurnal circadian rhytmety as basically depending on the signals received from Master Clock. Imbalance on external cues especially the light-dark cycle regulated by the light received from retino-hypothalamic tract, leads circadian disruption and misalignment on secretion of bodily hormones and enzymes which reveals serious metabolic consequences.

There present a study revealed the increasing BMI levels reasoned from long term increase in cortisol levels as a precursor of metabolic syndrome,obtained from hair sample of shiftworker subjects whose circadian timing has been disrupted due to the mismatch between external light and its normal bodily cycle. ${ }^{34}$ Increasing energy intake caused by misalignment on circadian timing caused by sleep restriction is another supposed peacemaker of obesity and circadian distruption. ${ }^{35}$ A recent study that has eliminated the effect of sleep restriction by comparing two groups those exposed to changing sleep timesand those having stable sleep times in addition to sleep restriction(5hours a day) on both groups in 8 consecutive days after 3 days of ten hour sleep to examine the metabolic consequences of circadian disruption have found decreasing insulin sensitivity and increasing inflammation as up to 2 times higher compared to start conditions for male participants in circadian misaligned group ${ }^{36}$ Fluxional metabolic rates over circadian disruption is another appointed intermediary conduce toward obesity. In the study that subjects underwent sleep restriction for 3 weeks and circadian disruption by changing the initial optimal sleephours at the baseline, declines in the resting metabolic rates and increase in the postprandial plasma glucose levels due to loss of pancreatic insulin secretion have been observed. ${ }^{37}$ In another study, the circadian rhytmcty measured by wrist temperature, has been found as valid predictor of future weight loss in response to diet intervention. To same study, low fragmentations and high amplitude of circadian rhytmcty was related with higher weight loss ensured by diet intervention. ${ }^{38}$
As mentioned before, food working through Gut $\mathrm{Clock}^{39}$ which is proposed as the other controller of satiety and hunger except Master clock in humans and also regulating the secretory peek periods of digestive system ${ }^{35}$ is another crucial external cue even can take place of light on the basis of regulation of Master clock to influence Slave clock thus peripheral tissue and organ functionings. By this view changing meal times appear as another causative agent of obesity due to imbalance between internal and external rhytmcty. In a study examine the effect of light at night on body weight, mice those exposed to dim or bright light during nighthours have been found as have increased body weight and impaired glucose tolerance compared to the those under the normal, 12hours light and 12hours dark cycle altough their daily caloric intake and physical activity levels did not differ in total. In addition, the observed difference among the meal timing of bright and dim light exposed mice(more food consumed during nighthours) compared the normal light cycle housing group proved the influence of meal timing disruption on weight gain. ${ }^{40}$ As cited in Verga et al..$^{35}$ the adipose tissue also has its own diurnal circadian rhytmcty also regulates secretions from adipocytes. Lipoprotein lipase, the enzyme responsible from fat digestion, reaches fever pitch after meal intake and if mismatch occurs between fat taken through the meal and lipoprotein lipase levels, due to the misaligned circadian clock of adipose tissue fat components are carried out and reveal toxic effect to other body structures particularly muscle and liver. So, the food consumption during the peak plasma levels of LPL which is also coincide with lighthours has been suggested. ${ }^{35}$

Molecular circadian clock: In addition to circadian rhythm regulated by external stimuli both central and peripheral clocks has diurnal rhytmicity controlled by 8 basic proteins, namely, CLOCK (circadian locomotor output cycle kaput), BMAL1 (brain and muscle ARNT like protein 1), two CRY (cryptochrome 1,2), three PER (period $1,2,3$ ) and REV-ERB, acting as negative or positive transational/ transcriptational feedback loops to maintain the circadian control of gene expressions. While the primary loop, BMAL1-CLOCK complex, act as positive loop by stimulating the transcription of PER and CRY, both REV-ERB and PER-CRY complex work as negative loop with their inhibitory actions. ${ }^{41,42}$ Altough the molecular circadian clock has its own rhytmycty regulated via the interaction of mentioned translational/transcriptational feedback loops also external cues like sleep and food availability can leads alterations on gene expressions. Previous human and murine studies have emphasized the impact of sleep on occcurance of unfavorable metabolic alterations reasoned by changing genetic expression of circadian clock. Completed researches on mice those mutated to disruption or removal of clock gene components illustrates the mediating role of circadian system on metabolic changes related with obesity. The pancreas related BMAL1 and CLOCK mutant mice have been reported as vulnerable to diabetes mellitus with reduced insulin secretion, impaired glucose tolerance and deformity of pancreatic beta cells accompanied by functional defects. ${ }^{43}$ Furthermore the pancreas specific BMAL1 knockout mice study has illustrated the role of BMAL1 on normal insulin secretion via ensuring normal beta cell functioning over preseving against circadian disruption and its protective role against accumulation of reactive oxygen species that thought as responsible from diabetes mellitus due to reduced glucose stimulated insulin secretion. ${ }^{44}$ Increasing CRY activity has been found beneficial for type 2 diabetes mellitus with enhancing fasting glucose levels and insulin sensitivity via its modulating role on transcriptional regulators of hepatic gluconeogenesis. ${ }^{45}$ In another study, that indicates the effect of clock proteins on regulation of circadian rhythm and metabolism, REV- 
ERB knockout mice have demonstrated altered circadian ryhytm and reduced audit on lipid metabolism. ${ }^{46}$ Besides, skeletal muscle specific BMAL1 knockout mice show impaired muscle glucose metabolism characterized by disrupted glucose stimulated insulin intake and reduced levels of insulin dependent glucose transporters. ${ }^{47}$

Changing energy expenditure and feeding times are thougt to as possible paths in terms of mediation between metabolic changes related with obesity and genes based circadian disruption. In a study examining the adipose tissue specific BMAL1 deprivation, the clock debarred mice became corpulant because of changing food intake times and increasing feeding induced by rising concentrations of polyunsaturated fatty acids(PUFA) secreted from adipocytes that regulate feeding by working as appetite regulator in hypothalamic neurons. ${ }^{48}$ In one of the recent study on circadian rhythm related gene in which the 721 subjects have CRY2 gene variants and 722 have MTNR1B gene variants were compared in terms of energy expenditure measurements, representing metabolic rate, and effect of preferred macronutrient pattern on those measures has examined via applying two year diet intervention, the CRY2 and MTNR1B genes have been found related with changes in energy expenditure measurements as response to two year diet intervention and dietary fat intake has found as indicator of the effect of those circadian rhythym related genes on detected changes on energy expenditure ${ }^{49}$ Additionally, recent studies also have emphasize the circadian rhytmicty of adipose related genes expressions as another potential link between obesity and circadian mechanism that has been found as inducing several metabolic syndrome components. ${ }^{50}$ By Gimble et al. ${ }^{51}$ the glucocorticoid induced leucine zipper, the gene expressed oscillations in terms of circadian biology of adipose tissue, has reviewed as one of the potential path between obesity and circadian contrivance because of its crucial roles on adipogenesis and osteogenesis. ${ }^{51}$ Hormonal changes also can be given as another incontrovertible obesity inducing manner resulted by suppressed expressions of circadian rhythm related genes. In a study, the human participants exposed to sleep deprivation for one night displayed the increase in amplitude of melatonin rhythm because of the suppression of BMAL1 expression ${ }^{52}$ which have been commentated as related with glucose metabolism in turn. ${ }^{41}$ Furthermore BMAL1 knockout mice have been shown as prone to obesity with reduced insulin sensitivity due to disrupted diurnal rhythm of insulin action. ${ }^{53}$ In the light of all these reviewed findings, the circadian mechanism seems to one of the vital impressive factor on obesity originating from sleep affairs (Table 1).

Table I Represent sleep deprivation induced circadian disruption and its obesity related outcomes

\begin{tabular}{llll}
\hline References & Sample number & Aim of the study & Results \\
\hline Leproult et al. ${ }^{36}$ & 26 healthy adults & $\begin{array}{l}\text { Determining adverse metabolic consequences of } \\
\text { sleep deprivation induced circadian misalignment }\end{array}$ & $\begin{array}{l}\text { Waned insulin sensitivity after sleep restriction both } \\
\text { in circadian aligned and misaligned groups Increased } \\
\text { inflammation and decreased insulin sensitivity in } \\
\text { circadian misaligned males as independent from } \\
\text { sleep deprival. }\end{array}$
\end{tabular}

$\begin{array}{lll}\begin{array}{l}\text { Ackerman et } \\ \text { al. }^{52}\end{array} & \text { I } 2 \text { young males } & \begin{array}{l}\text { Determining effects of sleep deprivation on circadian } \\ \text { mechanism }\end{array} \\ \text { Buxton et al. }{ }^{37} & \text { II young people } & \begin{array}{l}\text { Determining the relationship between sleep loss } \\ \text { combined circadian disruption and impaired glucose } \\ \text { metabolism and obesity. }\end{array} \\ \text { Marcheva et al. }{ }^{43} & 33 \text { shift workwers } & \begin{array}{l}\text { Determining the relationship between metabolic } \\ \text { syndrome and circadian disruption }\end{array} \\ & 89 \text { day workers } & \text {. }\end{array}$

Sleep loss induced BMALI supression Alterations on melatonin secretion rhytmicity and core peripheral circadian clock oscillators

Decreased resting metabolic rates

Decreased insulin secretion

Increased cortisol and BMI levels

\section{Sleep and hormones}

There present several studies have proved the reciprocal impact of sleep and hormones on each other. While the circadian rhythms and sleep variables like sleep quality and sleep cycles have impact on diurnal hormone regulation, hormones also can affect circadian rhytmcty and thereby sleep variables by working as internal cues regulating circadian system. To support, in a perivious meta analysis orexin has been reported as sleep wake cycle regulator ${ }^{54}$ in addition to its known role on appetite mechanism. As previously discussed each hormone has its own daily rhytmcty and flactuate with the influence of light and dark cycle and feeding behaviour as most important circadian system regulators. Kim et al..$^{55}$ has reported insulin resistance,obesity, hormonal and metabolic imbalance as considerable consequences of impaired hormonal rhythms induced by sleep impariment and sleep disturbances and hasrevieweddaily flactuation of sleep related hormones, namely, human growth hormone, TSH, leptin, ghrelin, melatonin and insulin as increasing level of GH during sleep, high melatonin levels in night, maximum level of TSH during middle of night and also maximum level of cortisol in middle of night with its peak level in the morning. ${ }^{55}$
Melatonin is a hormone secreted by pineal gland in response to darkness to prepare body for sleep and regulate waking and sleeping cycles. ${ }^{56}$ In a previous study,the daily intraperitonal melatonin injection for a week to 96hours sleep deprivated rats has been found as protective against memory impairment and oxidative stress in the brain, induced by sleep deprivation,on the occasion of role of melatonin on decreasing lipid and DNA peroxidation markers and also decreasing neural cell death ensured by its antioxidant and antiinflammatory effects on body. ${ }^{57}$ In another study, sleep restricted mice (4 hour sleep a day for eight experimental day) those demonstrate decreased glucose tolerance and HDL cholesterol levels despite of increased insulin resistance, total cholesterol and tryglicerides level have responded positively to intraperitonal melatonin and piromelatine, a melatonine agonist discoursing as beneficial on sleep maintenance and emotional disorders, in form of improved such metabolic dysfunctioning symptoms. ${ }^{58}$ Melatonin also has effects on carbohydrate metabolism via stimulating pancreatic glucagon secretion in addition to its inhibitory effect on insulin. ${ }^{59}$ Besides, detection of negative correlation between BMI score and urinary 6-HMS, a melatonin metabolite, has proved the role of melatonin on weight maintenance in addition to its negative relation with degree of sleep disorders also revealed 
by same study that conducted to examine the effect of melatonin suplemantation on weight control among postmenopausal women. ${ }^{60}$ Furthermore, in a study that reviewed melatonin to regulate glucose homeostasis via suppression of hepatic gluconeogenesis with activation of hypothalamic arc and enhance insulin resistance, nightly light exposure has been reported as impair glucose metabolism via repressed melatonin secretion that cause circadian disturbance. ${ }^{41}$ Due to the cited roles of melatonin on both sleep and weight maintenance, sleep curtailment in night conducing light exposure that display causative role on interruption of the nightly melatonin secretions give rise the potential role of melatonin on sleep loss induced obesity.

Human Growth Hormone secretions peak just after the sleep onset especially in NREM sleep. In a study, mice have been applicated growth hormone releasing hormone agonist showed beter performance in maze and thereby proves the research hypothesis that growth hormone therapy is beneficial in protection againt neurocognitive deficits leading by intermittent hypoxia as a cause of obstructive sleep apnea which is reported as related with suppression of growth hormone secretion during sleep. ${ }^{61}$ Additionally, in another previous study, growth hormone has diluted the detrimental effects of sleep deprivation on brain areas vulnerable to sleep disorders, especially hipocampus, with its role on prevising brain related cell proliferation. $^{62}$ In another previous study, growth hormone deficient children have exhibited reduced sleep time accompanied with reduced sleep efficiency. Growth hormone therapy also reported as has impact on sleep architecture in the same study ${ }^{63}$ as well as the influence of sleep stages on sleep related growth hormone secretion, such as deprivation on stage 3 which is also known as slow wave sleep, and stage 4 that lead supression of growth hormone peak during sleep. ${ }^{64}$ In one of the study represented the effects of sleep on growth hormone secretion, participants display monophasic growth hormone oscillation in the normal sleep condition (8.5hours a day) while in the sleep curtailment condition, in the form of 4.5 hours a day,biphasic oscillation of growth hormone secretion has been observed which extend the period of oscillation for 50minutes without any difference in total secreted amount compared to normal condition. Such change in nightly growth hormone secretion has been related with increased free fatty acid amount during sleep restriction. ${ }^{65}$ It has been reviewed that growth hormone defens towards hypoglycemia by acting as counter regulatory hormone against the hepatic and peripheral effects of insulin during sleep, leading extended time of fasting, to keep blood glucose level stable or ensure minimal fall through the agency of such aforementioned proven effect of growth hormone on free fatty acid release. ${ }^{66}$ On the strengths of highlighted direct metabolic effects of growth hormone and interralation with sleep, this hormone attracts attention in terms of sleep and obesity relation.

Both human and animal studies, have reported alterations on thyroid functions as one of the endocrine change subsequent to sleep restriction. In a cross-over study conducted with 15 healthy normal weight men those underwent to 2 consequent nigtht of sleep restriction and 2 normal sleep night respectively, increasing thyroxin (T4) and triiodothyronine (T3) levels accompanied with blunting nightly Tiroid Stimulating Hormone (TSH) have been reported in the condition of sleep restriction. ${ }^{67}$ Likewise, increased peripheral activity of T4 and T3 and central hypothyroidism has been observed in sleep deprivated wistar rats. ${ }^{68}$ As a contrary to such studies put forth increasing thyrothropic axis activity, in a recent study 14days exposure to sleep restriction ( 4.5 hour a day) hasresulted as decreased THS and T4 activity in females. ${ }^{69}$
Altough the thyroid function activity affected by sleep varies with sex and either the sleep restriction is acute or chronic, ${ }^{67}$ sleep related alterations in thyrothropic axis activity has found associated with glucose metabolism. In a study comparing the type 2 diabetic patients, and prediabetic patients, having impaired glucose tolerance, and healthy individuals in terms of thyroid hormones, increased levels of thyroid hormones has been observed in those with type 2 diabetes mellitus and impaired glucose tolerance. Increased insulin resistance has found as positively related with increased levels of thyroid hormones in the same set of experiment ${ }^{70}$ as consistent with the finding that patients with hypothyroidism and subclinical hypothyroidism have displayed higher insulin resistance and decreased insulin stimulated glucose transport compared to euthyroid subjects. ${ }^{71}$ As well as, the decreasing insulin stimulated glucose transport and rising insulin resistance have been also observed among patients with hyperthyroidism. ${ }^{72}$ Such findings indicate the thyrothropic axis activity as potential pathological mechanism lies under alterations on glucose metabolism.

Cortisol is a steroid hormone secreted by adrenal glands in response to stressfull conditions, morning awakening and physical activity in exercise level. ${ }^{73}$ This hormone has daily diurnal rhythm as rising in middle of night and reaching the peak level during morning awakeninghours ${ }^{55}$ Like that occurs in other hormones, sleep curtailments and detoriations disrupt the normal diurnal rhytmcty of cortisol that particularly results in elevated cortisol levels reasoned from increasing hypothalamic pitutiary adrenal axis(HPA) activity that induced by stress. In a study subjects exposed to four days of 4.5hours sleep a day, cortisol levels that been expected to peak during awakening, has reached their maximum 2hours earlier in addition to 23 percent higher levels in the evening and early night. ${ }^{65}$ As well as elevated evening and afternoon cortisol levels has been observed in participants subjected to sleep restriction ${ }^{74}$ as consistent with the finding that higher serum cortisol levels obtained from those exposed to sleep disruption. ${ }^{75}$ Such increasing levels of cortisol have detrimental effects as weight gain, impaired blood glucose levels, immune system supression, cardiovascular diseases and gastrointestinal problems ${ }^{73}$ due to its crucial role on glucose metabolism and energy regulation. In a study that has detect elevated levels of cortisol in response to acute sleep deprivation whereas decreased levels as a result of chronic circadian misalignment, rising plasma levels of pro-inflammatory and anti-inflammatory proteins namely, tumor necrosis factor alpha(TNF-a), interleukin-10(IL-10) and C-reactive protein(CRP) has been found as related with alterations on cortisol levels induced by sleep deprivation and circadian missalignment. ${ }^{76}$ In the study examined the patients with type 2 diabetes mellitus, elevated cortisol levels has been discovered as related with hig fasting plasma glucose, high cholesterol levels and high prevelance of ischemic heart disease. ${ }^{77}$ Elevated cortisol levels also has been reported as predictor of greater weight gain in hospitalized anorexic adolescents. ${ }^{78}$ In addition to crucial impacts of cortisol levels on body and metabolism, it also affects the sleep architecture and sleep duration. High morning cortisol levels has been recorded as negatively correlated with both nightly sleep duration and slow-wave sleep whereas it has revealed positive asssociation with stage 2 sleep $^{79}$ and also with stage 1 and REM sleep ${ }^{80}$ In another recent study, high morning cortisol levels has exhibited possitive correlation with insomnia symptoms in children. ${ }^{81}$ So, cortisol levels influence metabolism with the mediation of its role on both sleep duration and quality as well as its direct affects.

Recent researches has underlined adipokines, adipocyte derived 
hormones that have roles on regulation of insulin resistance, inflammation ${ }^{82}$ and also appetite and energy balance regulation mechanism, ${ }^{66}$ in terms of their sleep induced changes acting as matter for obesity and comorbid disorders. Alterations on adipokine secretions induced by sleep loss has been reported as create chronic sub-inflammatory state that leads impairment on glucose metabolism by causing insulin resistance and type 2 diabetes mellitus. ${ }^{83}$ Hayes et al. ${ }^{82}$ has found sleep curtailment as associated with increase in both leptin and visfatin levels, adipokines reported as related with inflammation and insulin resistance those being accepted as among biomarkers of obesity. Besides, in the same study each one hour reduction in REM sleep also has been found related with respectively $\% 15$ and $\% 31$ increase in leptin and visfatin levels.

Sleep duration has also been found to modify the circulating concentrations of orexigenic and anorexic hormones that play central role in appetite and energy regulation mechanism. In a previous study elevated leptin and decreased ghrelin levels has been observed in the subjects those demonstrated high BMI levels as a result of less sleep habits. ${ }^{17}$ In another study elevated ghrelin levels among participants subjected to acute sleep restriction has been related with increase in caloric intake, especially from sweets, snacks and carbohydrates with any alteration on leptin levels. ${ }^{84}$ Besides in the study that has compared obese and normal weighted adolescents in terms of changes in sleep loss induced metabolism-relevant hormones, orexin, leptin and ghrelin, and also BMI levels, among those sleep less than 8hours obese participiants exhibit lower sleep times than normal weighted subjects and leptin, ghrelin and orexin levels that accompanied with increased nightly snack intake were higher in both obese and normal weighted subjects those sleep less than 8hours which is considered as short for the age group of studied subjects(10-15yeaars).$^{85}$ Decrease in leptin and increase in ghrelin levels those lead increase in energy need and hunger respectively has been reported as a response of body to changing energy balance as elevated need for caloric intake that induced by increase in wake time due to sleep curtailment ${ }^{86}$ in contrast to the finding that 8 day sleep restricted subjects have demonstrate increased caloric intake compared to normal sleepers without any alteration on ghrelin and leptin levels or energy expenditure rates caused by sleep curtailment. ${ }^{86}$

In addition to those mentioned sleep related hormonal changes, both animal and human studies being completed in this field has suggested incontrovertible associations between sleep loss or impairments and problems with glucose metabolism.In a study it has been reported that healhty, normal weight participants had demonstrate reduction in glucose tolerance that caused by decrease in insulin sensitivity with elevated concentrations of $\mathrm{C}$ peptide those stated by elevated insulin and glucose peak response to breakfast accompanied with blunted glucagon responses after 2 days of sleep restriction as 4hours per night compared to 2 nights of regular sleep to 8 hours per night. ${ }^{87}$ Another study that subjected healthy men and women to 5 week of sleep restriction in conjunction with circadian misalignment as 5 hours of sleep per night with libitum food intake opportunity, has detected reduced intravenous and oral insulin sensitivity for both sexes as suggesting the detrimental effects of sleep impairments on glucose metabolism. ${ }^{88}$ In the study that Barf and covorkers have investigated the effects of acute and chronic sleep disturbance and sleep restriction on glucose homeostasis in rats, subjects demonstrate reduced insulin and increased glucose responses to intravenous glucose tolerance test (IVGT) without any difference between sleep restriction or sleep disturbance conditions. Besides, subjects exhibit hyperglycemia as a response to both sleep restriction and sleep disturbance with the difference that acute changes in sleep has not been found related with any alterations on insulin response while chronic conditions have caused such change. ${ }^{31}$ The reduced insulin secretion with no alteration on plasma glucose levels and hypothalamic insulin receptors has been reported in wistar rats that subjected to 4 days of sleep disturbance. ${ }^{89}$ The optimal glucose homeostasis has been observed for 7.5-8.5hours of sleep in the study that has revealed $u$-shaped relation between glucose homeostasis and total sleep duration. ${ }^{29}$ In contrast Byberg and covorkers have not pointed any relation between total sleep time and insulin secretion or insulin sensitivity besides to unchanged plasma fasting glucose levels. However they have reported such change as $2 \%$ increase in both periferal and hepatic insulin sensitivity and $1 \%$ decrease in insulin secretion for 1 hour increase in sleep quality rather than sleep duration. Additionally, in the same study, increase in sleep duration has been found related with reduced $\mathrm{HbAlc}$ levels as $0.3 \mathrm{mmol} / \mathrm{mol}$ decrease per each one hour increase in total sleep time and increase in sleep duration also has found related with $25 \%$ decrease in impairment of glucose metabolism in the measurements that have been adjusted for waist circuferences of subjects. ${ }^{33}$ In another previous study that participants exposed to 4days of sleep restriction, insulin sensitivity that measured by frequently sampled intravenous glucose test (FSIGT) has been reported as 23\% lower in sleep restricted participants than normal sleepers. The findings of the same study as quite high insulin and glucose responses given to breakfast and increased fasting plasma insulin levels that observed after sleep restriction proves the hypothesis that sleep restriction leads insulin resistance and diabetes mellitus. In addition to such shown impaired glucose metabolism, those mentioned alterations on insulin levels has been found correlated with increased free fatty acid levels which leads several obesity related metabolic impairments in turn. ${ }^{65}$ In consideration of all mentioned studies hormonal alterations appear as one of the important liable agent in the wake of relevance at isssue (Table 2).

Table 2 Represents sleep related hormonal alterations

\begin{tabular}{lll}
\hline Hormones & References & Outcomes \\
\hline Melatonin & ${\text { Tan et al. }{ }^{41}}^{\text {Broussard et al. }{ }^{65}}$ & $\begin{array}{l}\text { Supression of nocturnal melatonin secretion via exposure of light at night } \\
\text { and } 50 \text { minutes delay in oscillation as a result of sleep restriction that found to be related with increased } \\
\text { plasma free fatty acid levels. }\end{array}$ \\
& Rodrigues et al. ${ }^{65}$ & $\begin{array}{l}\text { Sleep deprivation led increased peripheral T3 and T4 activity and hypothiroidism in Wistar rats } \\
\text { TSH }\end{array}$ \\
& Schmid et al. ${ }^{67}$ & $\begin{array}{l}\text { Sleep restriction produced attenuated TSH secretion and increased T3 and T4 secretion in human } \\
\text { participants. }\end{array}$ \\
& Kessler et al. ${ }^{69}$ & Decreased TSH and T4 activity in female participants who underwent I4 days of sleep restriction
\end{tabular}


Table Continued....

\begin{tabular}{|c|c|c|}
\hline Hormones & References & Outcomes \\
\hline \multirow[b]{2}{*}{ Cortisol } & $\begin{array}{l}\text { Broussard et al.. }{ }^{65} \text { Wolkow } \\
\text { et al., }{ }^{74} \text { Song et al. }{ }^{75}\end{array}$ & Increased cortisol levels, perticularly at night, in sleep deprivated participants. \\
\hline & Wright et al. ${ }^{76}$ & $\begin{array}{l}\text { Sleep restriction and circadian misalignment induced flactuation in cortisol levels have found as related with } \\
\text { increase in TNF-alfa, IL-I0 and CRP levels }\end{array}$ \\
\hline \multirow{2}{*}{ Adipokines } & Hayes et al. ${ }^{82}$ & $\begin{array}{l}\text { Decrease in sleep hours has reported as related with increased leptin and visfatin levels those thought as } \\
\text { antecedents of obesity and insulin resistance. }\end{array}$ \\
\hline & Padilha et al. ${ }^{83}$ & $\begin{array}{l}\text { Impaired glucose metabolism and type } 2 \text { DM have reported as a result of alterations on TNF-alfa and IL-6 } \\
\text { that stemming from sleep restriction }\end{array}$ \\
\hline \multirow{4}{*}{$\begin{array}{l}\text { Leptin \& } \\
\text { Ghrelin }\end{array}$} & Broussard et al. ${ }^{65}$ & $\begin{array}{l}\text { Acute sleep restriction has resulted in stationary leptin levels but increased ghrelin levels that illustrated } \\
\text { positive association with increased caloric intake from deserts and snacks }\end{array}$ \\
\hline & Fue et al. ${ }^{85}$ & $\begin{array}{l}\text { Increased leptin and ghrelin levels in participants aged between } 10 \text { and } 15 \text { and sleep less than } 8 \text { hours at } \\
\text { night showed associations with snack intakes at nights }\end{array}$ \\
\hline & Calvin et al. ${ }^{86}$ & $\begin{array}{l}\text { Sleep restricted participants have demonstrated stationary leptin and grelin levels and energy expenditure } \\
\text { in spite of increased caloric intake }\end{array}$ \\
\hline & Taheri et al. & $\begin{array}{l}\text { After sleep restriction participants have demonstared weight gain with increased leptin and decreased } \\
\text { ghrelin levels. }\end{array}$ \\
\hline \multirow{3}{*}{ Insulin } & Eckel et al. ${ }^{88}$ & $\begin{array}{l}\text { Decreased oral and intravenous insulin sensitivity in human participants exposed to } 5 \text { hours of night sleep } \\
\text { for } 5 \text { weeks. }\end{array}$ \\
\hline & Koren et al. ${ }^{29}$ & $\begin{array}{l}\text { The U-shaped relationship has been detected between sleep duration and glucose metabolism and 7.5-8.5 } \\
\text { hours of sleep has been reported as optimal for ace glucose homeostasis. }\end{array}$ \\
\hline & Schmid et al. ${ }^{67}$ & $\begin{array}{l}\text { Sleep restriction in healthy normal weighted participants has resulted in increased C-peptide and insulin } \\
\text { levels and escalated glucose response to breakfast that arising from impaired glucose metabolism and insulin } \\
\text { resistance. }\end{array}$ \\
\hline
\end{tabular}

\section{Sleep and metabolism}

The extent of change in energy expenditure to contribute weight gain as a result of sleep restriction has been demonstrated by several researches with its possible paths as change in components of energy expenditure $^{90}$ increased fatigue and altered thermoregulation ${ }^{11}$ and increased opportunity of time to eat. ${ }^{91}$ Benedict et al. ${ }^{90}$ had reported $\% 5$ decrease in resting metabolic rate and \%20 decrease in postprandial energy expenditure after sleep deprivation in subjects exposed to one night total sleep deprivation and one night of regular sleep conditions in a crossover design. There were no difference in terms of caloric intake between conditions while decreased core body temperatures, fancied as long term weight gain contributor, after sleep deprivation has been reported. ${ }^{90}$ As it reversed, another crossover designed study that conducted with adolescents boyshas demonstrated negative energy balance after sleep deprivation conditions in two paths which are increased energy expenditure due to increased spontaneous physical activity accompanying increased wakefullnes and decreased caloric intake as a result of decreased motivation of eating. ${ }^{92}$ In a study has been conducted with 14 healthy young women, after 4 nightsof sleep restriction partcicipants has demonsrated net weight gain as a result of increased caloric intake contrariwise to stable energy expenditure components like resting energy expenditure (REE), thermic effect of food (TEF) and Physical activity levels (PAL). Additionally, sleep loss has been found as cause increased glucose induced thermogenesis (GIT), triiodothyronine (T3) and thyroxine (T4)levels as conversely related with the percentage of bodily fat mass whose high levels lead weight gain by inhibiting increase in thermogenesis. ${ }^{93}$ One of the recent study have indicated the role of increased food intake rather than alterations on energy metabolism in terms of short sleep induced weight gain among non-obese premenopousal healhty women those expressed stable postprandial thermogenesis and substrate oxidation after 3 consecutive night of 4 hours of sleep. ${ }^{94}$ Calvin et al. ${ }^{86}$ have reported stable activity energy expenditure (AEE),physical activity level (PAL) and circulating leptin and ghrelin levels in study subjects being exposed to 8 nights of sleep curtailment that has brought about 559 additional calories and resulted in 677 net incrase in caloric intake in comparison with normal sleep condition. ${ }^{86}$ In another study those underwent 4 hours of sleep condition have demonstared stable total energy expenditure (TEE) and resting metabolic rates (RMR) on the contrary intake of additional 300 calories especially from fat accompanying increased hunger and appetite rates compared with habitual 9 hour of sleep condition..$^{95}$ Insufficient sleep has been found to favor weight gain by increasing caloric intake especially after dinner, considered as inflicted by retardation on melatonin onset caused by circadian disruption, in spite of increased energy expenditure. ${ }^{96}$ As reviewed by Chaput et al..$^{91}$ rising energy intake reasoned from increased opportunity for time to eat accompanying short sleep times and rising levels of acitivities paired with the times expended as awake such as video games, television viewing, music listening and cognitive working that lead additional caloric intake increase the energy consumption while in another side energy expenditure tend to decrease as a result of low levels of physical activity including exercise and spontaneous daily phsical activity due to sleep loss induced fatigue and decrease in nonexercise activity thermogenesis that comprises motions like stance allocation and fidgeting etc. ${ }^{11}$ In a longitudinal study, sleep related decreasing physical activity levels with the mediation of too much television viewing has been reported as responsible from childhood obesity. ${ }^{97}$

\section{Sleep and neural changes}

In the study that subjects have been kept under 5.5 and 8.5 hours of nightly sleep conditions in random order, increased consumption of snacks that have high content of carbohydrate without any change in 
amount of meals has been indicated to favor obesogenic environment with stable energy expenditure components in short sleepers in laboratory conditions with add libitum food access. ${ }^{98}$ In the same way, less than 8hours of sleep have been found related with 475 or more additional calories those from snacks and tendency toward consumption of foods high in fat and low in carbohydrate content among adolescents. ${ }^{99}$

Sleep loss related such snacking behaviour forms the nonhomeostatic part of feeding that is stimulated by environmental, reward related, emotional and cognitive cues that processed in specific parts of brain mainly, striatum, some parts of pefrontal cortex and limbic system and explain the changes in food intake related with sleep restriction in addition to homeostatic changes in feeding behaviour including alterations on peripheral hunger and bodily hormones related with satiety and appetite. ${ }^{100,101}$ Recent studies discourse the effect of sleep loss on hedonic processes related to the some parts in the brain that enhance the drive to eat. In a study, total sleep deprivation led participants to show increased activation in right anterior cingulate cortex, the part of frontal cortex responsible from evaluation of the rewarding quality of food, in reply to food images as independent from caloric content in spite of same hunger ratings and same fasting plasma glucose before the scanning with those under normal sleep condition.Such finding emphasize the effect of prolonged wakefullness in terms of alterations on reward modulatory brain systems. ${ }^{102}$ In another study, participants underwent sleep restriction showed higher activation in insula, medial prefrontal cortex and orbitrofrontal cortex, the brain parts related with hedonic functions, in response to unhealthy foods in comparison with healthy foods. In the same study sleep restricted participants demonstrated tendency toward consuming high fat diet that thought to activating the brains dopaminergic system like sugary foods with the mediation of mesoaccumbens dopaminergic pathway ${ }^{103}$ that reported as highly activated in REM sleep. ${ }^{104}$ Baldo et al., ${ }^{100}$ has reported 10 days of sleep deprivation in rats as resulted in transcriptional changes in opioid striatal sytstem and hypothalamic energy-sensing system genes in the fashion of upregulation of proenkephalin(ENK), prodynorhin(DNY), seen combined in depressed mood, and Neuoropeptide Y(NPY) and downregulation of cocaine-and amphetamine-regulated transcript(CART) as favoring increase in palatable food intake and hedonic taste reactivity that brought answer to snacking behaviour coming with sleep loss. ${ }^{100}$

\section{Sleep, hypertension, cardiovascular disease and diabetes mellitus}

Several researches have indicated sleep duration as a risk factor for rising hypertension, type 2 diabetes mellitus and cardiovascular disease incidences. To a study conducted with urban African population, increased nightly sleep duration led increase in both systolic and diastolic blood pressure and after a certain level each hour of sleep led $\% 0.30$ and $\% 0.50$ decrease for systolic and diastolic blood pressure respectively in females, while only day time napping over a half hour lower the blood pressure in male participants. ${ }^{105}$ In the Nurses' Health Studies conducted with women between 1986 and 2000 five or less than fivehours of nightly sleep has been reported as related with increased risk for prevelance of hypertension with the mediation of high levels of BMI in participants younger than 60years. Such detected relation between hypertension prevelance and nightly short sleep duration that ruled out the 60years older participants have been interpreted as resulted by increased sympathetic nervous system activation due to sleep lossespecially for middle aged participants while the main reason of hypertension incidences in participants older than 60years is age related loss of arterial deference. ${ }^{106}$ In one of the recent research that detected U-shaped relationship between sleep duration and hypertension for all age and sex groups, high prevelances of hypertension have been reported among female those in age groups that younger than 45 and older than 65years and sleep less than 6hours and also in those aged between 45 and 65years and sleep 10 or morehours, while such increased risk have been recognized for male those sleep 10 or morehours at ages younger than 45 and sleep less than 6hours between ages of 45 and $65 .{ }^{107} \mathrm{In}$ addition to sleep restriction induced increase in sympathetic nervous system activity, increased salt intake and reduced renal salt-fluid excretion as a stress response to sleep restriction act as another possible path in terms of sleep related hypertension by creating chronic renal overload. ${ }^{108}$ Sleep loss related elevations of CRP levels ${ }^{76}$ also has been reported as related with increased hypertension prevelance. ${ }^{?}$

Perry et al. ${ }^{109}$ has reported sleep restriction related with cardiovascular impairment with the mediaton of increased renal sympathetic nervous system activity(rSNA) accompanying decrease in Angiotensin, ${ }^{1-7}$ a bioactive peptide act as a component of reninangiotensin system(RAS) and responsible from activation of peripheral vasodilator mechanisms by its stimulating effect on vasodilator prostaglandins and NO release. ${ }^{109}$ In another study, sleep restriction caused downregulation of gamma aminobutyric acid a receptors(GABAa) in paraventricular nucleus(PVN), the nuclei involve central neurons responsible from cardiovascular system control besides those in rostroventrolateral medulla(RVLM), have been found as responsible from increase in rSNA in rats as contributing cardiovascular alterations. ${ }^{110}$ Beside, in a previous research, each one hour increase in sleep duration has been reported as protective against cardiovascular disease with approximately $33 \%$ decrease in probability of coronary artery calsification that characterized with accumulation of calcified plaques to the arteries and act as future predictor of development of coronary diseases. ${ }^{111}$ As mentioned before, short sleep duration have been reported as related with impaired glucose metabolism and type 2 diabetes mellitus with the mediation of reduced glucose tolerance, ${ }^{87}$ reduced insulin sensitivity, ${ }^{88}$ hyperglycemia, ${ }^{32}$ and impaired glucose regulation. ${ }^{33}$ The possible paths mediating the relationship between type 2 diabetes mellitus and sleep loss has been reviewed as elevated evening cortisol levels as a stress response to sleep restriction that causing impaired glucose metabolism and inhibitory effect of increased sympathetic tone on pancreatic action. In addition, sleep loss related melatonin suppression have been reported as another mediator of increased sympathetic nervous activation that acting as the common pathology under sleep loss related hypertension, cardiovascular diseases and type 2 diabetes mellitus.

\section{Conclusion}

Human and animal studies demonstrates $\mathrm{u}$ shaped relationship between sleep and obesity, type 2 diabetes mellitus, impaired glucose metabolism, cardiovascular diseases, and hypertension those thought to be induced by sleep related impaired circadian rhythm, hormonal,metabolic and neural alterations as basic mechanisms. However, experimental and laboratory studies being conducted in this field, have revealed conflicting findings in terms of the extents of alterations on such potential explanatory mechanisms. Several researches indicates the altered parameters of hunger and appetite on the hormonal basis, particularly as increase in leptin and decrease in ghrelin ${ }^{17}$ while some others demonstrated antipodean ${ }^{66}$ or no alterations 
in both hormones ${ }^{87}$ altough increased caloric intake is common denominator for all. Similarly, findings about sleep induced changes in energy expenditure components show discrepancy in completed studies. While a previous study have found sleep loss as favoring weight gain with decrease in energy expenditure components despite of stable caloric intake ${ }^{102}$ in some others, stable energy expenditures and increased caloric intake of sleep restricted participants was indicated to beat a path for positive energy balance. ${ }^{93-95}$ In contrast, in another study sleep loss that accompanied with decrease in caloric consumption an increase in energy expenditure has been reported as favor negative energy balance..$^{2}$

As stated, researches had revealed conflicting results in terms of the extent and direction of functions of basic mechanisms underlying the relationship between sleep and mentioned health disorders. Referring to the literature,several factors play decisive role for the extent of the influence of sleep loss or disturbance on health disorders. ${ }^{112}$ In a study that participants underwent sleep restriction for 5 nights as 4 hour sleep per night, men have exhibited greater caloric intake than women and the differences have been detected between food patterns of African Americans and Whites as a result of sleep loss as supporting the sex and race differences. ${ }^{12}$ In the same way, the study being conducted with participants aged between 6-18years, while short sleep durations have been detected as a risk factor for obesity in girls for all age groups, such risk detected in boys aged between 13-18 and tend to decrease in boys those ages between 6 and 12years. ${ }^{113}$ Sleep architecture also plays an important role for sleep loss induced health outcomes. The effect of sleep restriction varies as depending on whether which stage of sleep is influenced from intervention. ${ }^{28}$ To recent studies, SWS have been related with glucose metabolism ${ }^{29}$ while stage 2 sleep have reported as associated with energy expenditure and taste related request for food ${ }^{28}$ and REM sleep also with appetite related hormones. ${ }^{30}$

Indicating the late sleep times as another determinant, Spaeth et al. ${ }^{114}$ found late bedtimes to space greater caloric intake during sleep restriction conditions ${ }^{12}$ as like another study found late bedtimes as strongly related with increased waist-hip circumferences, bodily fat deposition and BMI levels as independent from sleep duration. ${ }^{20}$ Furthermore, both genotype and phenotype variables also act as indicator for effects of sleep loss. Bandin et al. ${ }^{115}$ has reported circadian impairments in those have CLOCK $3111 \mathrm{C}$ allele that had found to related with obesity, while CLOCK $311 \mathrm{~T}$ allele carriers demonstared more vigorous circadian rhythm status. ${ }^{115}$ In another study conducted as two experimental sleep restriction condition as 5 night of 4 hours of sleep with 60days interval, while participants demonstarated different responses to sleep restriction in terms of weight gain and caloric intake, such responses have revealed stability across conditions on the individual basis as supporting the genetic and phenotype differences as important indicators for sleep loss related outcomes. ${ }^{114}$ Because sleep loss acts as stress factor that actuate the HPA axis activity as bodily response to stressful conditions, the evaluation of such reponse and sleep related emotional distress with the light of individual differences like sex,race, sleep pattern, genotype and phenotype will shed light on future studies.

\section{Acknowledgements}

None.

\section{Conflict of interest}

Author declares that there is no conflict of interest.

\section{References}

1. World Health Organization.

2. Ilhan Satman, TurdepDiyabet $\% 7.2$ Yeni DM \%32.3 Bilinen DM $\% 67.7$ BGT \%6.7 TURDEP-I: Türkiye'de Diabetes Mellitus, TURDEP Group: D. Care. 25:1551-1556.

3. Alkerwi A, Crichton GE, Hébert JR. Consumption of ready-made meals and increased risk of obesity: findings from the observation of cardiovascular risk factors in Luxemburg (ORISCAV-LUX). Br J Nutr. 2015;113(2):270-277.

4. Harris JL, Pomeranz JL, Lobstein T, et al. A crisis in the market place: how food marketing contributes to childhood obesity and what can be done. Annu Rev Public Health. 2009;30:211-225.

5. Remik A, Stern BL. Children's television advertising and brand choice: a laboratory experiment. Journal of Advertising. 1977;6(3):11-17.

6. Xiao Q, Arem HA, Moore SC, et al. Large prospective investigation of sleep duration, weight change and obesity in the NIH-ARP and health study cohort. Am J Epidemiol. 2013;178(11):1600-1610.

7. Nagai M, Hoshide S, Kario K. Sleep duration as a risk factor for cardiovascular disease: a review of recent literature. Curr Cardiol Rev. 2010;6(1):54-61.

8. Mc Knight Eily LR, Liu Y, et al. Wheat A, Unhealthy sleep related behaviours-12 states, 2009. MMWR Morb Mortal Wkly Rep. 2011;60(8):233-238.

9. Keyes KM, Maslowsky J, Hamilton A, et al. The great sleep recession: changes in sleep duration among US adolescents, 1991-2012. Pediatrics. 2015;135(3):460-468.

10. Coughlin JW, Smith MT. Sleep, obesity, and weight loss in adults: Is there a rationale for providing sleep interventions in the treatment of obesity. Inter Rev of Psychiat. 2014;26(2):177-188.

11. Graulet M, Ordovas JM, Madrid JA. The chronobiology, etiology and pathophysiology of obesity. Inter Jour of Obes. 2010;34(12):1667-1683.

12. Spaeth AM, Dinges DF, Goel N. Sex and race differences in caloric intake during sleep restriction in healthy adults. Am J Clin Nutr. 2014;100(2):559-566.

13. Parvaneh K, Poh BK, Hajifaraji M, et al. Sleep deprivation is related to obesity and low intake of energy and carbohydrates among working Iranian adults: a cross sectional study. Asia Pac J Clin Nutr. 2014;23(1):84-90.

14. Chaput JP, Lambert M, Gray Donald K. Short sleep duration is independently associated with overweight and obesity in Quebec children. Can J Public Health. 2011;102(5):369-374.

15. Hijorth MF, Quist JS, Andersen R, et al. Change in sleep duration and proposed dietary risk factors for obesity in Danish school children. Pediat Obes. 2014;9(6):156-159.

16. Spaeth AM, Dinges DF, Goel N. Effects of experimental sleep restriction on weight gain, caloric intake, and meal timing in healthy adults. Sleep. 2013;36(7):981-990.

17. Taheri S, Lin L, Austin D, et al. Short sleep duration is asociated with reduced leptin, elevated ghrelin, and increased body mass index. Plos Med. 2004;1(3):e62.

18. Neto JCR, Lira FS, Venancio DP, et al. Sleep deprivation affects inflammatory marker expression in adipose tissue. Lipids Health Dis. 2010;9:125.

19. Kant AK, Graubard BI. Association of self-reported sleep duration with eating behaviours of American adults NHANES 2005-2010. Am J Clin Nutr. 2014;100(3):938-947. 
20. Jarrin DC, McGrath JJ, Drake CL. Beyond sleep duration: distinct sleep dimensions are associated with obesity in children and adolescents. Int $J$ Obes (Lond). 2013;37(4):552-558.

21. Patel SR, Hayes AL, Blackwell T, et al. The association between sleep patterns and obesity in older adults. Int Jour Obes. 2014;38(9):11591164.

22. Graulet M, Abellen PG, Alburquerque Béjar JJ, et al. Timing of food intake predicts weight loss effectiveness. Int Jour Obes. 2013;137(4):604-611.

23. Baron KG, Reid KJ, Kern AS, et al. Role of sleep timing in caloric intake and BMI. Obesity. 2011;19(7):1374-1381.

24. Yaginuma S, Sakuraba K, Kadoya H, et al. Early bedtime associated with salutary breakfast intake in Japanese Nursery School children. International Medical Journal. 2015;22(1):30-32.

25. Kim NH, Shin DH, Kim HT, et al. Association between metabolic syndrome and inadequate sleep duration and skipping breakfast. Korean J Fam Med. 2015;36(6):273-277.

26. Sahlin C, Franklin KA, Stenlund H, et al. Sleep in women: Normal values for sleep stages and position and the effect of age, obesity, sleep apnea smoking, alcohol and hypertension. Sleep Med. 2009;10(9):1025-1030.

27. Tasali E, Leproult R, Ehrmann DA, et al. Slow wave sleep and the risk of type 2 diabetes in humans. Proc Natl Acad Sci USA. 2008;105(3):10441049.

28. Shechter A, O'Keeffe M, Roberts AL, et al. Alterations in sleep architecture in response to experimental sleep curtailment are associated with signs of positive energy balance. Am J Physiol Regul Integr Comp Physiol. 2012;303(9):883-889.

29. Koren D, Gallagher PR, Katz LEL, et al. Sleep architecture and glucose and insulin homeostasis in obese adolescents. Diabetes Care. 2011;34(11):2442-2447.

30. Koban M, Le WW, Hoffman GE. Changes in hypothalamic corticotropinreleasing hormone, neuropeptide-Y and proopiomelanocortin gene expression during chronic REM sleep deprivation in rats. Endocrinology. 2006;147(1):421-431.

31. Barf RP, Meerlo P, Scheurink AJ. Chronic sleep disturbance impairs glucose homeostasis in rats. Int J Endocrinol. 2010;2010:819414.

32. Wang Y, Carreras A, Lee S, et al. Chronic sleep fragmentation promotes obesity in young adult mice. Obesity. 2014;22(3):758-762.

33. Byberg S, Hansen ALS, Christensen DL, et al. Sleep duration and sleep quality are associated differently with alterations of glucose homeostasis Diabet Med. 2012;29(9):354-360.

34. Manenschijn L, van Kruysbergen RG, de Jong FH, et al. Shift work at young age is associated with elevated long-term cortisol levels and body mass index. J Clin Endocrinol Metab. 2011;96(11):1862-1865.

35. Verga S, Tramuto M. Circadian rhythms and nutrition. Acta Medica Mediterranea. 2014;30:441-445.

36. Leproult R, Holmback U, Van Cauter E. Circadian misalignment augments markers of insulin resistance and inflammation, independently of sleep loss. Diabetes. 2014;63(6):1860-1869.

37. Buxton DM, Cain SW, O'Connor SP, et al. Adverse metabolic consequences in humans of prolonged sleep restriction combined with circadian disruption. Sci Transl Med. 2012;4(129):129-143.

38. Bandin C, Nicolas Martinez A, Ordovás JM, et al. Circadian rhytmicity as a predictor of weight-loss effectiveness. Int J Obes. 2014;38(8):10831088 .

39. Betchold DA. Energy Responsive time keeping. J Genet. 2008;87(5):447458 .
40. Fonken LK, Workman JL, Walton JC, et al. Light at night increases body mass by shifting the time of food intake. Proc Natl Acad Sci USA 2010;107(43):18664-18669.

41. Tan E, Scott EM. Circadian rhythms, insulin action and glucose homeostasis. Curr Opin Nutr Metab Care. 2014;17(4):343-348.

42. Johnston JD. Physiological links between circadian rhythms, metabolism and nutrition. Exp Physiol. 2014;99(9):1133-1137.

43. Marcheva B, Ramsey KM, Buhr ED, et al. Disruption of the clock components CLOCK and BMAL1 leads to hypoinsulinemia and diabetes. Nature. 2010;466(7306):627-631.

44. Lee J, Moulik M, Fang Z, et al. Bmall and beta-cell clock are required for adaptation to circadian disruption, and their loss of function leads to oxidative stress-induced beta cell failure in mice. Mol Cell Biol. 2013;33(11):2327-2338

45. Zhang EE, Liu Y, Dentin R, et al. Cryptochrome mediates circadian regulation of cAMP signalling and hepatic gluconeogenesis. Nat Med. 2010;16(10):1152-1156.

46. Cho H, Zhao X, Hatori M, et al. Regulation of circadian behaviour and metabolism by REV-ERB-alpha and REV-ERB-beta. Nature. 2012;485(7396):123-127.

47. Dyar KA, Cicilot S, Wright L, et al. Muscle insulin sensitivity and glucose metabolism are controlled by the intrinsic muscle clock. Mol Metab. 2014;3(1):29-41.

48. Paschos GK, Ibrahim S, Song WL, et al. Obesity in mice with adipocytespecific deletion of clock component Arntl. Nat Med. 2012;18(12):17681777.

49. Mirzaei K, Xu M, Qi Q, et al. Variants in glucose- and circadian rhythmrelated genes affect the response of enegy expenditure to weight-loss diets: the POUNDS LOST Trial. Am J Clin Nutr. 2014;99(2):392-399.

50. Gomez Abellan P, Hernandez Morante JJ, et al. Clock genes are implicated in the human metabolic syndrome. Int Jour Obes. 2008;32(1):121-128.

51. Gimble JM, Ptitsyn AA, Goh BC, et al. Delta sleep-inducing peptide and glucocorticoid-induced leucine zipper:potential links between circadian mechanism and obesity. Obes Rev. 2009;10(Suppl 2):46-51.

52. Ackerman K, Plomp R, Lao O, et al. Effect of sleep deprivation on rhythms of clock gene expression and melatonin in humans. Chronobiol Int. 2013;30(7):901-909.

53. Shi S, Ansari TS, McGuinness OP, et al. Circadian disruption leads to insulin resistance and obesity. Curr Biol. 2013;23(5):372-381.

54. Sakurai T, Mieda M, Tsujino N. The orexin system: roles in sleep/wake regulation. Ann N Y Acad Sci. 2010;1200:149-161.

55. Kim TW, Jeong JH, Hong SC. The impact of sleep and circadian disturbance on hormones and metabolism. Int Jour Endoc. 2015;2015:591729.

56. Yolende SE. Melatonin, Sleep and Insomnia. New York, USA: Nova Science; 2010

57. Kwon KJ, Lee EJ, Kim MK, J et al. The potential role of melatonin on sleep deprivation-induced cognitive impairment: implication of FMRP on cognitive function. Neuroscience. 2015;301:403-414.

58. She M, Hu X, Su Z, et al. Piromelatine, a novel melatonin receptor agonist, stabilizes metabolic profiles and ameliorates insulin resistance in chronic sleep restricted rats. European Journal of Pharmacology. 2014;727:60-65.

59. Bahr I, Mühlbaver E, Schucht H, et al. Melatonin stimulates glucagon secretion in vitro and in vivo. J Pineal Res. 2011;50(3):36-344. 
60. Ewa WK, Grazyna K, Chojnacki J, et al. The effect of melatonin supplemantation on the quality of sleep and weight status in postmenopausal women. Prz Menopauzalny. 2014;13(6):334-338.

61. Nair D, Ramesh V, Li RC, et al. Growth hormone releasing hormone (GHRH) signalling modulates intermittent hypoxia-induced oxidative stress and cognitive deficits in mice. Journal of Neurochemistry. 2013;127(4):531-540.

62. Garcia Garcia F, Herran DL, Juárez Aguilar E, et al. Growth hormone improves hipocampal adult cell survival and counteracts the inhibitory effect of prolonged sleep deprivation on cell proliferation. Brain Res Bull. 2011;84(3):252-257.

63. Verrilo E, Bizzarri C, Bruni O, et al. Effects of replacement therapy on sleep architecture in children with growth hormone deficiency. Sleep Med. 2012;13(5):496-502.

64. Drucker Colin RR, Spanis CW, Hunyadi J, et al. Growth hormone effects on sleep and wakefulness in rats. Neuroendocrinology. 1975;18:1-8.

65. Broussard JL, Chapotot F, Abraham V, et al. Sleep restriction increases free fatty acids in healthy men. Diabetologia. 2015;158(4):791-798.

66. Garcia Garcia F, Juarez Aguilar E, Santiago García J, et al. Ghrelin and its interactions with-growth hormone,leptin and orexins: implications fort he sleep-wake cycle and metabolism. Sleep Med Rev. 2014;18(1):89-97.

67. Schmid SM, Hallschmid M, Jauch Chara K, et al. Partial sleep restriction modulates secretory activity of thyrotropic axis in healthy men. $J$ Sleep Res. 2013;22(2):166-169.

68. Rodrigues NC, da Cruz NS, da Paula N, et al. Sleep derivation alters thyroid hormone economy in rats. Experimental Physiology. 2015;100(2):193-202.

69. Kessler L, Nedeltcheva A, Imperial J, Penev PD. Changes in serum TSH and T4 during human sleep restriction. Sleep. 2010;33(8):1115-1118.

70. Lambadiari V, Mitrou P, Maratoue E. Thyroid hormones are positively associated with insulin resistance early in the development of type 2 diabetes. Endocrine. 2011;39(1):28-32.

71. Maratou E, Hadjidakis DJ, Kollias A, et al. Studies of insulin resistance in patients with clinical and subclinical hypothyroidism. Eur J Endocrinol. 2009;160(5):785-790.

72. Maratou E, Hadjidakis DJ, Peppa M, et al. Studies of insulin resistance in patients with clinical and subclinical hyperthyroidism. Eur J Endecrinol. 2010;163(4):625-630.

73. Aronson D. Cortsiol-Its role in stress, inflammation, and indications for diet theraphy. Today's Dietitian. 2009;11(11):38.

74. Wolkow A, Aisbett B, Reynolds J, et al. The impact of sleep restriction while performing stimlated physical firefighting work on cortisol and heart rate responses. Inter Archiv of Occup and Envir Health. 2016;89(3):461-475.

75. Song HT, Sun XY. Effects of sleep deprivation on serum cortisol level and mental health in servicemen. Inter J Psychophysiol. 2015;96(3):169175 .

76. Wright KP, Drake AL, Frey DJ, et al. Influence of sleep deprivation and circadian misalignment on cortisol, inflammatory markers and cytokine balance. Brain Behav and Immun. 2015;47:24-34.

77. Reynolds RM, Labad J, Strachan MW, et al. Elevated fasting plasma cortisol is associated with ischemic heart disease and its risk factors in people with type 2 diabetes: The Eddinburgh Type 2 Diabetes Study. Jour of Clin Endocrinol. 2010;95(4):1602-1608.

78. Garber AK, Hetnel K. Elevated cortisol predicts greater weight gain in longitudinal study of adolescents hospitalized with anorexia nervosa. $J$ of Adoles Health. 2009;44(2):19.
79. Lemola S, Perkinson Gloor N, Hagmann von Arx P, et al. Morning cortisol secretion in school age children is related to sleep pattern of the preceding night. Psychoneuroendocrinology. 2015;52:297-301.

80. Hatzinger M, Brand S, Perren S, et al. In pre-school children, sleep objectively assessed via sleep-EEGs remains stable over 12 months and is related to psychological functioning, but not to cortisol secretion. $J$ Psychiatr Res. 2013;47(11):1809-1814.

81. Fernandez Mendoza J, Vgontsaz AN, Calhoun SL, et al. Insomnia symptoms, objective sleep duration and hypothalamic-pituitary-adrenal activity in children. Eur J Clin Invest. 2014;44(5):493-500.

82. Hayes AL, Xu F. Sleep duration and ciculating adipokine levels. Sleep. 2011;34(2):147-152.

83. Padilha HG, Zimberg IZ, De Souza DA, et al. A link between sleep loss, glucose metabolism and adipokines. Braz J Med Biol Res. 2011;44(10):992-999.

84. Broussard JL, Kilkus JM, Delebecque F, et al. Elevated ghrelin preditcts food intake during experimental sleep restriction. Obesity. 2016;24(1):132-138.

85. Fu JF, Zhou F, Xu XQ, et al. Short sleep duration as a risk factor for obesity in childhood is associated with increased leptin, ghrelin and orexin levels. HK J Pediatr. 2013;18:152-158.

86. Calvin AD, Carter RE, Adachi T, et al. Effects of experimental sleep restriction on caloric intake and activity energy expenditure. Chest. 2013;144(1):79-86.

87. Schmid SM, Hallschmid M, Jauch Chara K, et al. Disturbed glucoregulatory response to food intake after moderate sleep restriction. Sleep. 2011;34(3):371-377.

88. Eckel RH, Depner CM, Perreault L, et al. Morning circadian misalignment during short sleep duration impacts insulin sensitivity. Curr Biol. 2015;25(22):3004-3010.

89. Moraes AD, Venancio DP, Suchecki D. Sleep deprivation alters energy homeostasis through non-compensatory alterations in hypothalamic insulin receptors in Wistar rats. Horm Behav. 2014;66(5):705-712.

90. Benedict C, Hallschmid M, Lassen A, et al. Acute sleep deprivatin reduces energy expenditure i healthy men. Am J Clin Nutr. 2011;93(6):12291236.

91. Chaput JP, Klingenberg L. Do all sedentary activities lead to weight gain: sleep does not. Curr Opin Clin Nutr Metab Care. 2010;13(6):601-607.

92. Klingenberg L, Chaput JP, Holmbäck U, et al. Sleep restriction is not associated with a positive energy balance in adolescent boys. Am J Clin Nutr. 2012;96(2):240-248.

93. Bosy Westhpal A, Hinrichs S, Jauch Chara K, et al. Influence of partial sleep deprivation on energy balance and insulin sensitivity in healthy women. Obesity Facts. 2008;1(5):266-273.

94. Shechter A, Rising R, Wolfe S, et al. Postprandial thermogenesis and substrate oxidation are unaffected by sleep restriction. Int Jour Obes. 2014;38(9):1153-1158.

95. St Onge MP, Roberts AL, Chen J, et al. Short sleep duration increases energy intakes but does not change energy expenditure in normal-weight individuals. Am J Clin Nutr. 2011;94(2):410-416.

96. Markwald RR, Melanson EL, Smith MR, et al. Impact of insufficient sleep on total daily energy expenditure, food intake, and weight gain. PNAS. 2013;110(14):5695-5700.

97. Magee C, Caputi P, Iverson D. Lack of sleep could increase obesity in children and too much television could be partly to blame. Acta Pediatr. 2013;103(1):e27-e31. 
98. Nedeltcheva AV, Kilkus JM, Imperial J, et al. Sleep curtailment is accompanied by increased intake of calories from snacks. Am J Clin Nutr. 2009;89(1):126-133.

99. Weiss A, Xu F, Storfer Isser A, et al. The association of sleep duration with adolescents' fat and carbohydrate consumption. Sleep. 2010;33(9):12011209.

100. Baldo BA, Hanion EC, Obermeyer W, et al. Upregulation of gene expression in reward-modulatory striatal opioid systems by sleep loss Neuropsychopharmacology. 2013;38(13):2578-2587.

101. Berthoud H. Homeostatic and non-homeostatic pathways involved in the control of food intake and energy balance. Obesity. 2006;14(Suppl 5):197-200.

102. Benedict C, Brooks SJ, O’Daly OG, et al. Acute sleep deprivation enhances the brain's response to hedonic food stimuli: an fMRI study. $J$ Clin Endocrinol Metab. 2012;97(3):443-447.

103. St Onge MP, Wolfe S, Sy M, et al. Sleep restriction increases the neuronal response to unhealthy food in normal-weight individuals. Inter Jour of Obes. 2014;38(3):411-416

104. Murray G, Nicholas CL, Kleiman J, et al. Nature's clocks and human mood: the circadian system modulates reward motivation. Emotion. 2009;9(5):705-716

105. Pretorius S, Stewart S, Carrington MJ, et al. Is there an association between sleeping patterns and other environmental factors with obesity and blood pressure in an urban African population. Plos One. 2015;10(10):e0131081.

106. Gangswich JE, Feskanich D, Malaspina D, et al. Sleep duration and risk for hypertension in women: results from the Nurses' Health Study. Am J of Hyper. 2013;26(7):903-911.
107. Fang J, Wheaton AG, Keenan NL, et al. Association of sleep duration and hypertension among US adults varies by age and sex. Am J Hypertens. 2012;25(3):335-341.

108. Folkow B. Mental stress and its importance for cardiovascular disorders; physiological aspects,' from-mice-to-man'. Scand Cardiovasc J. 2001;35(3):163-172.

109. Perry JC, Bergamaschi CT, Campos RR, et al. Differential sympathetic activation induced by intermittent hypoxia and sleep loss in rats:Action of angiotensin(1-7). Auton Neurosci. 2011;160(1-2):32-36.

110. Perry JC, Bergamaschı CT, Campos RR, et al. Interconnectivity of sympathetic and sleep networks is mediated through reduction of gamma aminobutyric acidergic inhibition in the paraventricular nucleus. $J$ Sleep Res. 2014;23(2):168-175.

111. King CR, Knutson KL, Rathouz PJ, et al. Short sleep duration and incident coronary artery calcification. JAMA. 2008;300(24):2859-2866.

112. Cedernaes J, Schiöth HB. Determinants of shortened, disrupted and mistimed sleep and associated metabolic health consequences in healthy humans. Diabetes. 2015;64(4):1073-1080.

113. Cao M, Zhu Y, He B, et al. Association between sleep duration and obesity is age-and gender-dependent in Chinese urban children aged 6-18 years: a cross sectional study. BMC Public Health. 2015;15:1029-1039.

114. Spaeth AM, Dinges DF, Goel N. Phenotypic vulnerability of energy balance responsees to sleep loss in healthy adults. Sci Rep. 2015;8:1-6.

115. Bandin C, Martinez Nicolas A, Ordovas JM, et al. Differences in circadien rhytmicity in CLOCK $3111 \mathrm{~T} / \mathrm{C}$ genetic variants in moderate obese women as assessed by thermometry, actimetry and body position. Int J Obes. 2013;37(8):1044-1050. 